msh-mss Mathématiques et sciences humaines

193 | Printemps 2011

Varia

\title{
Introduction au numéro
}

Foreword

Jean-Pierre Desclés

\section{OpenEdition}

Journals

Édition électronique

URL : http://journals.openedition.org/msh/11943

DOI : $10.4000 /$ msh. 11943

ISSN : $1950-682$

\section{Éditeur}

Centre d'analyse et de mathématique sociales de l'EHESS

\section{Édition imprimée}

Date de publication : 15 janvier 2011

Pagination : $5-6$

ISBN : 09876936

ISSN : 0987-6936

\section{Référence électronique}

Jean-Pierre Desclés, «Introduction au numéro », Mathématiques et sciences humaines [En ligne], 193 Printemps 2011, mis en ligne le 01 juin 2011, consulté le 23 juillet 2020. URL : http:// journals.openedition.org/msh/11943 


\title{
INTRODUCTION
}

\author{
Jean-Pierre DESCLÉS
}

Marc Barbut est certainement l'un des mathématiciens qui, avec Georges Théodore Guilbaud (EHESS) et André Lentin (Université René Descartes), ont su et ont réussi magistralement à développer le champ de recherches dans les «mathématiques, sciences humaines et sociales ». Les intérêts de Marc Barbut pour les structures mathématiques (structures d'ordres par exemple), les mesures (probabilités) et l'analyse des données dans sciences humaines (économie, psychologie, sociologie, ethnologie, linguistique) sont multiples et variées.

Directeur d'études à l'EHESS, directeur du CAMS (Centre d'Analyse Mathématiques Appliquées aux sciences sociales) de l'EHESS pendant de nombreuses années, Marc Barbut a organisé des enseignements de mathématiques à la Sorbonne pour les psychologues et les sociologues, il a organisé l'UFR de mathématiques à l'université René Descartes (Paris V) puis il a organisé le département de mathématiques appliquées aux sciences humaines à l'université de Paris-Sorbonne (Paris IV) qui a été intégré à l'Institut des Sciences Humaines Appliquées (ISHA) de cette même université. Il a dirigé pendant de nombreuses années l'ISHA, devenu une UFR qui regroupe des mathématiciens, des informaticiens, des sociologues et des linguistes. Ses travaux ont connu une grande audience au-delà des frontières de la France, en particulier en Espagne.

Marc Barbut a su définir et expérimenter des voies pédagogiques pour enseigner les mathématiques à des étudiants de sciences humaines, non seulement à la Sorbonne mais aussi à l'EHESS dans la structure expérimentale de l'EPRASS (Enseignement Préparatoire Appliquées aux Sciences Sociales) qui fut une véritable réussite, tant sur le plan pédagogique qu'institutionnel. Ses cours, ses livres d'enseignement, ses articles ont formé d'un côté, des chercheurs en sciences humaines et sociales en leur apprenant comment utiliser adéquatement les mathématiques et les statistiques, en leur apprenant à se méfier d'utilisation mécaniques et sans comprendre de techniques mathématiques et d'un autre côté, ils ont appris aux étudiants en mathématiques à comprendre comment les mathématiques pouvaient pénétrer dans les domaines des études portant sur l'homme et les sociétés en partant de problèmes posés par ces domaines.

Ses collègues et amis de l'Université René Descartes, de l'Université de ParisSorbonne, de l'EHESS, de l'ISHA et du CAMS, d'universités espagnoles sont heureux de présenter ici quelques unes de leurs contributions scientifiques, exposées à la Journée «Mathématiques et Sciences humaines ${ }^{1}$, dans ce champ interdisciplinaire mathématiques, informatique, sciences humaines, sciences sociales en essayant de suivre le chemin que Marc Barbut a ouvert.

\footnotetext{
${ }^{1}$ Dans le cadre du Colloque Épistémologie des sciences humaines, 17-19 décembre 2009.
} 


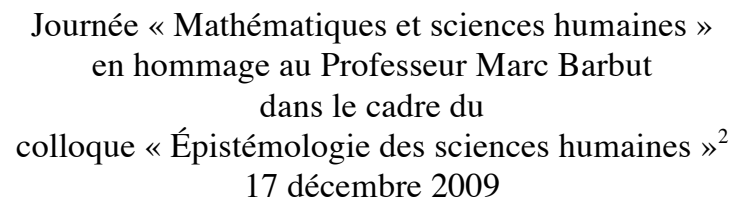

Sylvain Auroux (Université Paris Diderot, LHTL)

Introduction

Bernard Valade (Université Paris Descartes, GEPECS)

La mesure des inégalités : Marc Barbut critique de Vilfredo Pareto

Pierre Parlebas (Université Paris Descartes, GEPECS)

Modélisation mathématique, jeux sportifs et sciences sociales

Bertrand Saint-Sernin

Articulation entre savoir mathématique et décision administrative

Thierry Martin (Université Franche Comté, Laboratoire Logique de l'agir)

Gabriel Tarde et la statistique criminelle

Michel Bourdeau (EHESS, IHPST)

L'idée de mathématique appliquée chez Comte

Alain Degenne (EHESS, CMH)

À propos de la notion de rôle dans l'analyse des relations sociales

Alain Kirman (Université Aix-Marseille III, GREQAM)

Marc Barbut, Marseille et Pareto

José Arribas (UNED, Madrid)

Marc Barbut et l'Espagne

Bruno Leclerc et Bernard Monjardet (EHESS, CAMS)

Structure d'ordres et sciences humaines

Jean-Pierre Desclés (Université Paris-Sorbonne, LaLIC)

Les sciences humaines ont-elles besoin de mathématiques?

\footnotetext{
${ }^{2}$ Organisé par l'École doctorale « Concepts et langages », l’UFR ISHA de l'Université Paris-Sorbonne et le GEPECS de 1'Université Paris Descartes.
} 